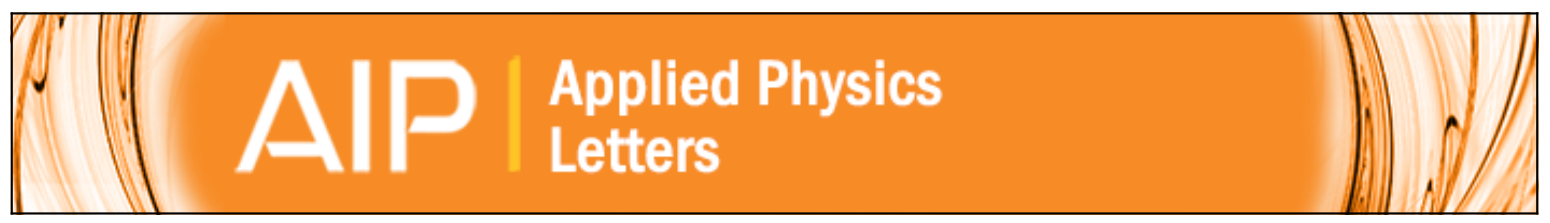

\title{
High-frequency behavior of the Datta-Das spin transistor
}

H. López, X. Oriols, J. Suñé, and X. Cartoixà

Citation: Applied Physics Letters 93, 193502 (2008); doi: 10.1063/1.3021073

View online: http://dx.doi.org/10.1063/1.3021073

View Table of Contents: http://scitation.aip.org/content/aip/journal/apl/93/19?ver=pdfcov

Published by the AIP Publishing

$\underset{\substack{\text { Powbonsug } \\ \text { R }}}{A}$ Re-register for Table of Content Alerts

\section{Create a profile.}




\title{
High-frequency behavior of the Datta-Das spin transistor
}

\author{
H. López, X. Oriols, J. Suñé, and X. Cartoixà ${ }^{a)}$ \\ Departament d'Enginyeria Electrònica, Universitat Autònoma de Barcelona, 08193 Bellaterra, Spain
}

(Received 10 August 2008; accepted 20 October 2008; published online 10 November 2008)

\begin{abstract}
We have studied the high-frequency behavior and the ultimate limiting factor to the cutoff frequency for the Datta-Das spin transistor using device Monte Carlo simulations. We have found that the maximum frequency of operation is not related to intrinsic parameters to the spin of the carriers, such as the Larmor frequency or the spin lifetime, but to the transit time through the channel. (C) 2008 American Institute of Physics. [DOI: 10.1063/1.3021073]
\end{abstract}

Spintronics ${ }^{1-3}$ has been of great interest in recent years as it has been gradually realized that one scarcely used quantum property of the electron, i.e. its spin, can be put to work for information processing, transmission, and storage. Metalbased spintronics can already be found in commercial devices such as the giant magnetoresistance-based ${ }^{4}$ hard drive read heads, magnetic random access memories, or highspeed isolators. Semiconductor-based spintronics is not yet as mature, but the degree of control achievable with semiconductor heterostructures together with the prospects of high-speed low-power logic associated with switching spins instead of displacing charge makes it an attractive topic.

One of the best known device proposals in semiconductor spintronics is the spin analog of the electro-optic modulator, ${ }^{5}$ which has come to be known as the Datta-Das spin transistor (DDST). In this device the average spin of an ensemble of electrons is manipulated through a gate bias by means of the Rashba effect, ${ }^{6}$ and its relative alignment with respect to the magnetization of the collector will determine whether a high or a low current is measured. While this proposal has stimulated a vast amount of research, it has never come to be realized experimentally; the difficulties ranging from the conductance mismatch problem, ${ }^{7,8}$ which hinders spin injection from ferromagnets into semiconductors, to the need for a narrow two-dimensional electron gas (2DEG) channel (a quantum wire is optimal) to reduce spin relaxation, ${ }^{9,10}$ or the need for ballistic operation-although recent reports suggest that nonballistic operation of the DDST might be possible with no change ${ }^{11}$ or under slightly different operating principles. ${ }^{12,13}$

It is reasonable to expect that the maximum operating frequency theoretically attainable by the DDST is controlled by the average transit time through the device. However, one can envisage a situation with a perfectly spin selective source and drain initially in the "on" state, with all spins aligned with both source and drain, where a short pulse reversing all spins is applied: after a time scale related to the spin precession time, no more electrons will exit through the drain and the device will be in the "off" state. Of course, this simple argument does not take into account the effects of the displacement current, but it serves to illustrate the appropriateness of studying more closely the factors limiting the highfrequency behavior in order to discard more complicated dynamics.

In this letter we resort to a time-dependent Monte Carlo (MC) simulation of the DDST in order to investigate this

\footnotetext{
${ }^{a)}$ Electronic mail: xavier.cartoixa@uab.es.
}

issue. There have been previous MC studies of the DattaDas transistor, ${ }^{9,11,14,15}$ and while the effects of an ac gate on a 2DEG channel with Rashba field have been thoroughly analyzed, ${ }^{16}$ systematic studies of the ac performance of the DDST have been missing so far.

For the MC simulation of the DDST we have considered a device with planar geometry and electronic motion confined in the $x-y$ plane (see Fig. 1), i.e., a 2DEG. The device consists of a ferromagnetic (FM) source and drain (contacts) and a semiconductor high mobility channel bridging them. The channel is surrounded by air and reflecting boundary conditions have been employed at the channel/air interfaces.

The device simulator is based on the widely used device $\mathrm{MC}$ technique ${ }^{17}$ with the electrons inside the device moving according to the semiclassical equations of motion. For twodimensional systems, the subband of the semiclassical electron is accounted for in the MC formalism only at injection time. The electron is injected at a $z$ according to the probability density for the corresponding subband and is not allowed to change $z$ thereafter (except after some intersubband scattering event, not considered here). The confinement energy will just be an offset that is subtracted from the total electron energy before the remaining energy is assigned to motion along the allowed directions. Because of the small dimension of the device, electrons are made to move through the channel only under the influence of an applied voltage $(V)$ and the Coulomb interaction among them $\left(V_{e e}\right)$, computed from the self-consistent solution to the Poisson equation. In our case, spin has been added as an additional three-component vector attached to each electron. This methodology has already been used for the simulation of spintronic devices. ${ }^{18}$

Thus, electrons are allowed to move freely in the 2DEG according to the Hamiltonian

$$
H=\frac{\mathbf{p}^{2}}{2 m^{*}}+V+V_{e e}+H_{\text {spin }},
$$

where $\mathbf{p}$ is the electron momentum and $m^{*}$ is the effective mass, including a contribution from the Rashba spin

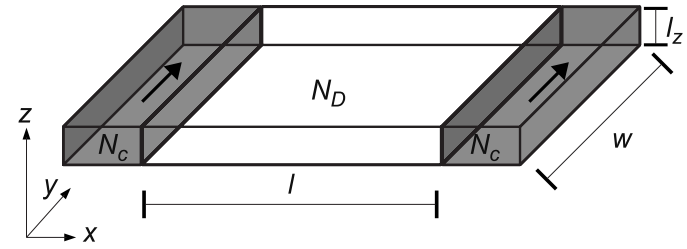

FIG. 1. Device geometry showing the choice of axes and the different lengths involved in the simulation. 
Hamiltonian $^{6}$ with an additional term describing the effect of the gate ac modulation, which dictates the spin evolution,

$$
\begin{aligned}
H_{\text {spin }}(\mathbf{k}, t) & =\left[\alpha_{\mathrm{dc}}+\alpha_{\mathrm{ac}} \cos \omega t\right]\left(\sigma_{x} k_{y}+\sigma_{y} k_{x}\right) \\
& =\hbar \boldsymbol{\Omega}(\mathbf{k}, t) \cdot \sigma,
\end{aligned}
$$

where $\alpha_{\mathrm{dc}}$ is the Rashba coefficient coming from the dc part of the gate bias, $\alpha_{\mathrm{ac}}$ is the amplitude of the modulation to the Rashba coefficient due to the gate bias oscillating at a frequency $\omega, \mathbf{k}$ is the crystal momentum of the electron under consideration, $\sigma$ are the Pauli spin matrices, and $\boldsymbol{\Omega}(\mathbf{k}, t)$ is a local momentum- and time-dependent Larmor precession vector after a convenient rewriting of the terms.

As we are considering ballistic transport, we will not include any scattering events other than those with the lateral boundaries and the cases where the electron is prevented from leaving the device due to the partially transmitting contact, and spins will only evolve unitarily under the action of the Rashba field. Therefore, our simulations take into account a spin relaxation mechanism closely related to the D'yakonov-Perel ${ }^{19,20}$ (DP) one, although scattering off boundaries can sometimes cause a different behavior of the spin ensemble. ${ }^{21}$ This mechanism is acknowledged as the predominant one in $n$-doped 2DEGs. ${ }^{22}$

Since the electrical spin injection and detection from FM contacts can be thought of in terms of a spin-dependent contact resistance, ${ }^{23,24}$ we have modified ${ }^{25}$ a recently published ${ }^{26}$ charge injection algorithm for ohmic contacts in ballistic devices to allow for a nonunity probability of injection/ extraction into/from the active region, effectively capturing the physics of the spin-dependent contact resistances (see Ref. 25 for details). Also, seeing that we are interested in the ultimate intrinsic factors that limit the high-frequency operation, we will not consider the effect of spurious capacitances due to the device geometry, which can severely impact the maximum operating frequency. ${ }^{27}$ The instantaneous current was computed using the Ramo-Shockley theorem, ${ }^{28}$ which takes into account particle and displacement current.

The simulated semiconductor is GaAs with effective mass $m^{*}=0.063 m_{0}$, where $m_{0}$ is the free electron mass, and lattice temperature $T=300 \mathrm{~K}$. The active region has a doping $N_{D}=10^{17} \mathrm{~cm}^{-3}$, while in the contacts the Fermi energy was set to $E_{f}=0.01 \mathrm{eV}$, which corresponds to a doping $N_{c}$ $\approx 1.4 \times 10^{18} \mathrm{~cm}^{-3}$. All results shown correspond to a channel length $l=20 \mathrm{~nm}$, a width $w=50 \mathrm{~nm}$, and a confinement height $l_{z}=10 \mathrm{~nm}$. The energy difference between the first two conduction subbands is $\sim 0.18 \mathrm{eV}$, much larger than the other energies available to the system; and therefore only the first subband has been considered in the simulations.

Since we intend to perform the calculations in the regime of linear operation of the device and yet have a strong enough signal allowing us to discriminate between the on (low resistance) and off (high resistance) states above the noise, we first obtain the source-to-drain $I-V$ characteristics for the FM contacts either with their magnetizations aligned [parallel (P)] or antialigned [antiparallel (AP)]. The results are shown in Fig. 2, and they provide us with an upper bound to the current difference, which we would be able to obtain in the ideal case that all spins arrived at the drain exactly reversed. The solid line in Fig. 2 is the result of a prediction considering separate contact resistances for each spin channel, ${ }^{25}$ from which we extract that significant deviations from linearity will occur for voltages above $40 \mathrm{mV}$ For the

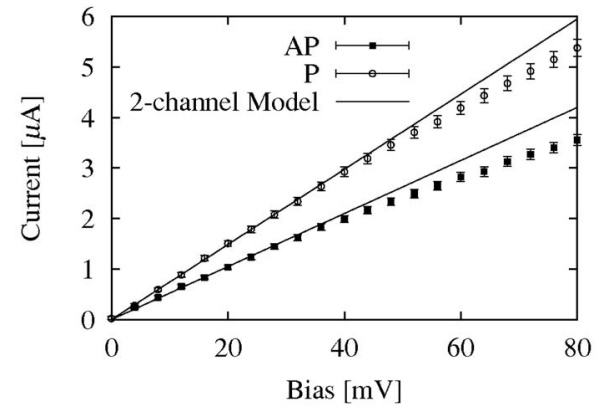

FIG. 2. I- $V$ characteristics of the device without Rashba coupling, corresponding to the $\mathrm{P}$ and AP configurations of the magnetization in the FM contacts. Error bars signal one standard deviation in the average. The solid line corresponds to a linear regime simplified model prediction (cf. Ref. 25).

dc results, the current was calculated by a long average of instantaneous currents after the steady state was reached.

In order to select the range $\left[\alpha_{\mathrm{dc}}-\alpha_{\mathrm{ac}}, \alpha_{\mathrm{dc}}+\alpha_{\mathrm{ac}}\right]$ of operation, we plot in Fig. 3 the source-to-drain currents as a function of $\alpha_{\mathrm{dc}}$ for $\alpha_{\mathrm{ac}}=0$ for different source voltages. We observe that for large $\alpha_{\mathrm{dc}}$ 's, the sensitivity to variations in the Rashba factor decreases. This is expected, as a larger $\alpha_{\mathrm{dc}}$ will reduce the spin diffusion length and, therefore, will cause the spins to reach the collector with a higher degree of randomization. We select the range $\alpha=\left[12 \times 10^{-10}, 36\right.$ $\left.\times 10^{-10}\right] \mathrm{eV} \mathrm{cm}$ at $30 \mathrm{mV}$ since it ensures a roughly linear response to variations in $\alpha_{\mathrm{dc}}$ and it is a range that should be achievable with the proper combination of material system $^{29,30}$ and the application of external gate biases. ${ }^{31,32}$ In the following we will refer to this range as R1.

We now proceed to simulate the dynamic response of the source-to-drain current for several modulating frequencies for the range R1, obtaining the plots shown in Fig. 4. As it can be seen, the system progressively becomes unable to follow the modulation as the frequency increases. The normalized amplitudes of the source-to-drain time-dependent current for several modulating frequencies are shown in Fig. 5 , from where we extract a cutoff frequency of $7.2 \mathrm{THz}$ for the DDST operating in R1. For these plots, shown after steady state was reached, an average for each time over different realizations was performed so that a sufficient signalto-noise ratio was obtained. This is equivalent to performing a single simulation for a longer time in order to obtain more clearly defined peaks in the Fourier transform of the current, while ensuring that transient effects have faded out.

Now, since we are interested in identifying whether the transit time or the spin precession frequency-averaged over all electrons-is the determining factor for the cutoff frequency, we perform a second set of simulations for a different range of spin-orbit strength, $\alpha=\left[70 \times 10^{-10}, 95\right.$

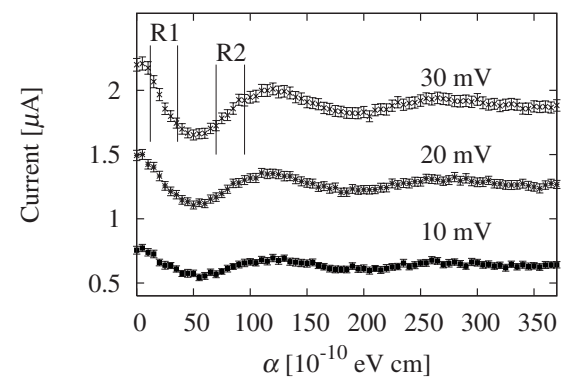

FIG. 3. Source-to-drain current as a function of the Rashba constant for several source bias. 


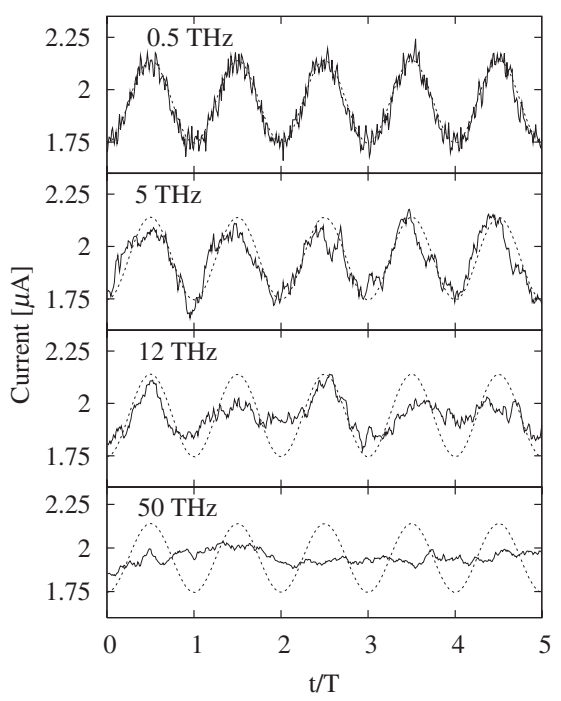

FIG. 4. Source-to-drain current as a function of time for several modulation frequencies $\omega$ for $\mathrm{R} 1$. The dotted line corresponds to the response in the quasistatic approximation, extracted from Fig. 3.

$\left.\times 10^{-10}\right] \mathrm{eV} \mathrm{cm}$, at $30 \mathrm{mV}(\mathrm{R} 2$ in Fig. 3). These values are probably very hard to achieve experimentally because of the large electric fields that should be present transverse to the channel, but we use them nevertheless as a way of increasing the spin precession frequency without substantially modifying the mean electron transit time. This is indeed achieved, obtaining for R2 an average precession frequency 3.4 times higher than in R1, but a similar transit time (57 fs for R1 and $67 \mathrm{fs}$ for R2). The variation in transit times under the two operating ranges is due to the spins arriving at the collector with different orientation in average, which changes the barrier transmittivity and, therefore, changes the number of carriers in the channel, which affects the motion of other carriers through $V_{e e}$.

We proceed to obtain from the R2 set of calculations (R2 curve in Fig. 5) a cutoff frequency of $6.1 \mathrm{THz}$. The ratio of cutoff frequencies is similar to the ratio of the inverses of the transit times, and very different from the ratio of precession frequencies which, in addition, predict an increase in the cutoff frequency instead of the observed reduction. This indicates that, similarly to charge transfer devices, the transit time is the factor determining the maximum intrinsic operating frequency of the present ballistic DDST. This is not surprising, as electrons spending several modulation periods inside the device will only respond to an average value of the Rashba field, not displaying any current modulation. Thus, we believe that this should be a general feature of the DDSTs.

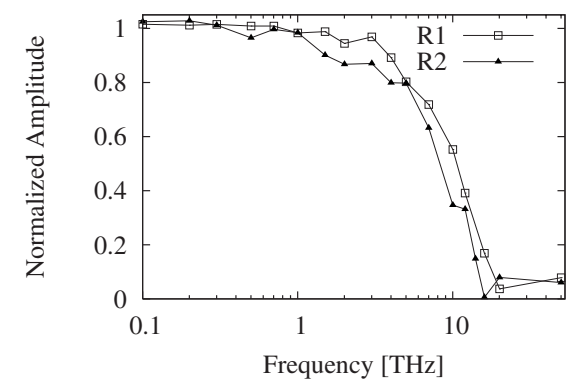

FIG. 5. Amplitude of the source-to-drain current for several Rashba modulating frequencies. A cutoff frequency of $7.2 \mathrm{THz}(6.1 \mathrm{THz})$ is observed for the range $\mathrm{R} 1$ (R2).
In summary, we have performed time-dependent MC simulations of a ballistic DDST under the action of an ac gate bias. We observe that the transit time of the electrons inside the channel, and not the Larmor frequency of the precessing spins around the Rashba field, is the limiting factor when determining the maximum intrinsic operating frequency.

This work was supported in part by the European Commission's Marie Curie International Reintegration under Grant No. MIRG-CT-2005-017198 and by the Spanish Ministry of Science and Technology under Contract No. TEC2006-13731-C02-01/02. J.S. acknowledges the support of the DURSI of the Generalitat de Catalunya. X.C. acknowledges the financial support from Spain's Ministry of Education and Science Ramón y Cajal program.

${ }^{1}$ G. A. Prinz, Phys. Today 48(4), 58 (1995).

${ }^{2}$ S. A. Wolf, D. D. Awschalom, R. A. Buhrman, J. M. Daughton, S. von Molnár, M. L. Roukes, A. Y. Chtchelkanova, and D. M. Treger, Science 294, 1488 (2001).

${ }_{3}^{3}$ I. Žutić, J. Fabian, and S. Das Sarma, Rev. Mod. Phys. 76, 323 (2004).

${ }^{4}$ M. N. Baibich, J. M. Broto, A. Fert, F. N. Vandau, F. Petroff, P. Eitenne, G. Creuzet, A. Friederich, and J. Chazelas, Phys. Rev. Lett. 61, 2472 (1988).

${ }^{5}$ S. Datta and B. Das, Appl. Phys. Lett. 56, 665 (1990).

${ }^{6}$ Y. A. Bychkov and E. I. Rashba, J. Phys. C 17, 6039 (1984).

${ }^{7}$ G. Schmidt, D. Ferrand, L. W. Molenkamp, A. T. Filip, and B. J. van Wees, Phys. Rev. B 62, R4790 (2000).

${ }^{8}$ J. Fabian, A. Matos-Abiague, C. Ertler, P. Stano, and I. Žutić, Acta Phys. Slov. 57, 565 (2007).

${ }^{9}$ A. A. Kiselev and K. W. Kim, Phys. Rev. B 61, 13115 (2000).

${ }^{10}$ A. W. Holleitner, V. Sih, R. C. Myers, A. C. Gossard, and D. D. Awschalom, Phys. Rev. Lett. 97, 036805 (2006).

${ }^{11}$ M. Ohno and K. Yoh, Phys. Rev. B 77, 045323 (2008).

${ }^{12}$ X. Cartoixà, D. Z.-Y. Ting, and Y.-C. Chang, Appl. Phys. Lett. 83, 1462 (2003).

${ }^{13}$ J. Schliemann, J. C. Egues, and D. Loss, Phys. Rev. Lett. 90, 146801 (2003).

${ }^{14}$ M. Shen, S. Saikin, and M.-C. Cheng, IEEE Trans. NanoTechnol. 4, 40 (2005).

${ }^{15}$ M. Ohno and K. Yoh, Phys. Rev. B 75, 241308 (2007).

${ }^{16}$ C.-S. Tang, Int. J. Mod. Phys. B 20, 869 (2006).

${ }^{17}$ C. Jacoboni and P. Lugli, The Monte Carlo Method for Semiconductor Device Simulation (Springer, Berlin, 1989).

${ }^{18}$ S. Saikin, Y. V. Pershin, and V. Privman, IEE Proc.: Circuits Devices Syst. 152, 366 (2005).

${ }^{19}$ M. I. D'yakonov and V. I. Perel', Fiz. Tverd. Tela (S.-Peterburg) 13, 3581 (1971) [Sov. Phys. Solid State 13, 3023 (1972)].

${ }^{20}$ M. I. D'yakonov and V. Y. Kachorovskiı̌, Fiz. Tekh. Poluprovodn. (S.Peterburg) 20, 178 (1986) [Sov. Phys. Semicond. 20, 110 (1986)].

${ }^{21}$ C.-H. Chang, A. G. Mal'shukov, and K. A. Chao, Phys. Rev. B 70, 245309 (2004).

${ }^{22}$ W. J. H. Leyland, G. H. John, R. T. Harley, M. M. Glazov, E. L. Ivchenko, D. A. Ritchie, I. Farrer, A. J. Shields, and M. Henini, Phys. Rev. B 75, 165309 (2007).

${ }^{23}$ E. I. Rashba, Phys. Rev. B 62, R16267 (2000).

${ }^{24}$ D. L. Smith and R. N. Silver, Phys. Rev. B 64, 045323 (2001).

${ }^{25}$ H. López, X. Oriols, J. Suñé, and X. Cartoixà, J. Appl. Phys. 104, 073702 (2008).

${ }^{26}$ X. Oriols, E. Fernàndez-Díaz, A. Alvarez, and A. Alarcón, Solid-State Electron. 51, 306 (2007).

${ }^{27}$ S. Bandyopadhyay and M. Cahay, Appl. Phys. Lett. 85, 1433 (2004).

${ }^{28}$ V. Gruzinskis, S. Kersulis, and A. Reklaitis, Semicond. Sci. Technol. 6, 602 (1991).

${ }^{29}$ X. Cartoixà, D. Z.-Y. Ting, E. S. Daniel, and T. C. McGill, Superlattices Microstruct. 30, 309 (2001).

${ }^{30}$ X. Cartoixà, D. Z.-Y. Ting, and T. C. McGill, J. Comput. Electron. 1, 141 (2002).

${ }^{31}$ J. Nitta, T. Akazaki, H. Takayanagi, and T. Enoki, Phys. Rev. Lett. 78, 1335 (1997)

${ }^{32}$ T. Schäpers, G. Engels, J. Lange, T. Klocke, M. Hollfelder, and H. Lüth, J. Appl. Phys. 83, 4324 (1998). 